\title{
.
}

\section{Lung Cancer Risk Perception Among Older Smokers; a Cross-sectional Study}

\author{
Fatemeh Zarghami (iD) ${ }^{1,2,3}$, Hamid Allahverdipour ${ }^{4}$ and Mohammad Asghari Jafarabadi (iD ${ }^{2,5,{ }^{*}}$ \\ ${ }^{1}$ Iran's National Elites Foundation, Center For International Science and Technology Cooperation (CISTC), Tehran, Iran \\ ${ }^{2}$ Department of Biostatistics and Epidemiology, Tabriz University of Medical Sciences, Tabriz, Iran \\ ${ }^{3}$ Department of Population Health Sciences, Virginia Polytechnic Institute and State University, Blacksburg, USA \\ ${ }^{4}$ Department of Health Education and Health Promotion, Faculty of Health, Tabriz University of Medical Sciences, Tabriz, Iran \\ ${ }^{5}$ Road Traffic Injury Research Center, Tabriz University of Medical Sciences, Tabriz, Iran \\ "Corresponding author: Department of Biostatistics and Epidemiology, Faculty of Health, Tabriz University of Medical Sciences, P.O. Box: 5166614711, Tabriz, Iran. Tel: \\ +98-4133357580 (385), Fax: +98-4133340634, Email: m.asghari862@gmail.com
}

Received 2020 February 01; Revised 2020 November 14; Accepted 2020 November 14.

\section{Abstract}

Background: Lung cancer is one of the deadliest cancers around the world. Also, scientific evidence proves the considerably lower lung cancer risk perceptions of smokers compared to non-smokers.

Objectives: This study aimed at assessing the risk perception of smokers about their risk to contract lung cancer and their perceptions about lung cancer survival.

Methods: In this cross-sectional study, the data were collected from 215 smokers in Tabriz, Iran, between April 2019 and July 2019. The data collection tool was designed, using validated questionnaires and contained questions on the perceived risk of smokers to develop lung cancer and their perception of lung cancer survival. A general linear model was conducted to model the relationship of risk perception with underlying predictors considering a significance level of 0.1.

Results: The results of modeling indicated that perceived susceptibility (P-Sus) was negatively related to "years smoked" in simple regression $(\mathrm{B}=-0.035, \mathrm{P}=0.069)$ and multiple regression $(\mathrm{B}=-0.069, \mathrm{P}=0.007)$. P-Sus was also negatively related to the variable "age" in the age category of 45 to 50 years $(B=-1.981, P=0.046)$. Moreover, perceived severity ( $\mathrm{P}-\mathrm{Sev})$ was negatively related to the variable "sex" $(\mathrm{B}=-1.037, \mathrm{P}=0.068)$. The results of the Spearman correlation indicated significant and direct correlations between "quitting intention" and P-Sus ( $r=0.296, \mathrm{P}<0.001), \mathrm{P}-\mathrm{Sev}(\mathrm{r}=0.162, \mathrm{P}=0.009)$.

Conclusions: Lung cancer risk perception of participants was moderately above the average and significant correlations were observed between "quitting intention" and "P-Sus" and "P-Sev" and "Risk Perception score". This could be utilized in developing educational programs to promote awareness about lung cancer, especially for smokers.

Keywords: Lung Cancer, Smokers, Regression Analysis, Health Risk Behaviors

\section{Background}

The second most prevalent cancer in men and women is lung cancer, which is also one of the deadliest cancer types (1). The number of death caused by lung cancer is more than the colon, breast, and prostate cancer combined each year (2). Despite advances in lung cancer treatment and management, the 5-year survival rate (2009 2015) of lung cancer is still very low at only $19.4 \%$ (3). Recent estimates show that almost one-third of the world population smokes (4). Smoking is responsible for increasing the prevalence of various health conditions such as cancers and the deaths resulting from these conditions (5).

Besides, the main proportion of cancer deaths and the cost of productivity loss associated with smoking is related to lung cancer (5). According to the results of a review article by Moosazadeh et al. (6) one-fifth of Iranian men and $2 \%$ to $3 \%$ of women have daily smoking habits. The findings of the Moosazadeh et al.'s (6) meta-analysis revealed that a large portion of the general population over the age of 15, and one-fifth of Iranian adult males smoke. Medical research has established much scientific evidence that smoking leads to lung cancer (7). Lung cancer is a fatal disease and, therefore, is the dominant cause of cancer death around the world (7). Active cigarette smoking is the preeminent cause of lung cancer besides many other identified lung cancer risk factors $(8,9)$. In this study, we examined the most common variable to assess smoking behaviors, pack-year (smoking of at least 1 pack per day in the last 
5 years), and other variables that affect the lifetime risk for lung cancer (age, number of years smoked, and frequency or packs of cigarettes smoked).

\subsection{Lung Cancer Risk Perception}

One of the factors affecting the process of decision making based on risk perception is based on an individual's frame of reference developed over a lifetime (10). Many health risks are the result of decisions made by individuals consciously trying to select the best possible choices for themselves and those important to them (11). Some of these choices are personal, such as wearing bicycle helmets and seatbelts, and following safety warnings (11). In other cases, the effects of individual choices are minor but accumulate over time (11). The result of the literature review provided us the evidence that smokers have a significantly lower risk perception of lung cancer compared to people, who do not smoke (12). The cognitive appraisal process of "threat appraisal" will be initiated in response to the fear-arousing health messages (if you smoke or continue to smoke, you will develop lung cancer) (13). Individuals consider two aspects of the perceived threat; one is the severity and the other one is susceptibility (13). Severity appraisals imply determining the level of harm that is expected from the specific threat (e.g. 'lung cancer can kill me.'). Susceptibility appraisals discuss how likely individuals are affected by the threat (e.g. 'because I am a smoker, I will develop lung cancer in my lifetime.') (13). Perceived lung cancer risk influences people to take action that could lead to a positive behavior change and in this instance, this action is quitting smoking (14). In line with tobacco control, numerous activities performed at the local, national, and international levels are emphasizing on behavior change by using health communication strategies and behavior change theories (10). Effective risk communication depends not only on presenting general risk factors and preventive information but also on factors that are specific to the individual (10).

\section{Objectives}

In this study, the perception of the smokers about their risk of developing lung cancer in their lifetime, their chance to survive if they develop lung cancer, and the relationship with quitting intention were assessed. After conducting a comprehensive literature review, it was identified that there has not been any study, if any, to evaluate the lung cancer risk perception among smokers, which affects important individual factors in making decisions to quit smoking. Therefore, this study aimed at determining lung cancer risk perception among smokers. The results could be used by future researchers or public health professionals in designing and implementing successful smoking cessation programs.

\section{Methods}

\subsection{Participants and Procedures}

This project is a cross-sectional study with a survey as a data collection tool and a sample size of 215 individuals. Smokers were defined as individuals with a smoking history of at least 1 pack per day in the past 5 years. The inclusion age criterion for the study was 45 years and over because lung cancer usually occurs in older adults. Most of the patients diagnosed with lung cancer are 65 years or older, while a very small percentage of them are younger than 45 years (15). The age range of lung cancer diagnosis is between 64 and 70 years (15). Peto (16) has concluded that the age of 40 is the youngest age, at which lung cancer is common to be the subject of studying". Therefore, the age 45 and over have been chosen as the inclusion age criteria for this study.

The Institutional Review Board of the Tabriz University of Medical Sciences granted permission to conduct the study (IRB ethical code: IR.TBZMED.REC.1397.955). The survey was distributed among potential participants between April 2019 and July 2019 in the parks, bus stations, retirement houses, senior health organizations, Pulmonology Clinic of Imam Reza, and Razi Hospital. The authors would like to note that the reporting of this manuscript was constructed based on strengthening the reporting of observational studies in epidemiology (STROBE) (17).

\subsection{Data Sources/Measurement}

The survey questions were adapted from validated surveys both in Persian and English languages, available in the scientific literature on the topic of lung cancer risk perception in general $(18,19)$. The final survey was designed to evaluate our target population's perception of lung cancer risk. These questions were modified and changed based on the objectives of this study and cultural adaptations were also applied. The survey was checked for reliability and construct validity. When English questions were translated into the Persian language, the survey was tested by the method of forward-backward translation. A highly experienced team of lung cancer and health education experts (10 individuals) reviewed the final Persian version of the questionnaire and confirmed its content and face validity 
in both quantitative and qualitative aspects. After this process, their comments were reviewed by the research team and the necessary modifications were applied to the questions (20). The final survey that was distributed among participants was in Persian and included questions on demographic information, smoking history, and perception of lung cancer risk (perception of developing lung cancer in lifetime and lung cancer survival).

The demographic characteristics of the participants (Table 1) were measured with the checklist designed based on the validated surveys with cultural adaptations. Three variables (education, occupation, and income) were used to create a new variable "score for socio-economic status" (SES score ). Firstly, each of the variables of education, occupation, and income was transformed into a scale of 1 to 6 . For instance, for the variable "occupation", the "office employee" and "receptionist" were given the same score, and "university faculty" and "teacher" were also given the same score. A combination of these new scores (1-6) for three variables (education, occupation, and income) was used to create a variable "SES level", which is measured on a scale of 1 to 3 (SES level: low, medium, and high). The questions to measure the lung cancer risk perception are designed on the scale of " 0 " to " 10 ", which were considered as the main outcome. The first variable was measuring "perceived susceptibility", (P-Sus) and was on the scale of "0" to "10":

If you continue to smoke, what is your chance of developing lung cancer? Please choose a number from " 0 " to "10", with "0" having zero chance of developing lung cancer and " 10 " having a definite chance of lung cancer.

The next variable is measuring "Perceived severity" (PSev) and is on the scale of " 0 " to " 10 ":

If you continue to smoke and develop lung cancer, what is your chance of being alive after 5 years? Please choose a number from " 0 " to " 10 ", with " 0 " having zero chance of surviving lung cancer and "10" having a definite chance of surviving lung cancer (definite survival).

The variables P-Sus and P-Sev were designed referring to studies conducted by Wong and Cappella (21) and Kotz et al. (22). Then, they were combined to create a new variable "Risk Perception score", which was measured on the scale of " 0 " to " 20 ". We also selected covariates to test for inclusion in our study based on the existing literature on risk perception (23). These included demographic characteristics presented in Table 1. Quitting intention of the participants was measured on a scale of 1 to 4 , with " 1 " the lowest intention and " 4 " the highest intention:

Which of the following statements describes your current situation best?

1) I smoke and I have no intention to quit in the next 6

\begin{tabular}{|c|c|}
\hline & No. (\%) \\
\hline \multicolumn{2}{|l|}{ SES level* } \\
\hline Low & $55(25.6)$ \\
\hline Medium & $124(57.7)$ \\
\hline High & $36(16.7)$ \\
\hline \multicolumn{2}{|l|}{ Marital status } \\
\hline Single & $8(3.7)$ \\
\hline Married-engaged & 188() 87.4 \\
\hline Divorced & $7(3.3)$ \\
\hline Widow & $12(5.6)$ \\
\hline \multicolumn{2}{|l|}{ Age, $y$} \\
\hline $45-50$ & $72(33.5)$ \\
\hline $51-55$ & $43(20.0)$ \\
\hline $56-60$ & $38(17.7)$ \\
\hline $61-65$ & $29(13.5)$ \\
\hline $66-70$ & $10(4.7)$ \\
\hline $71-75$ & $14(6.5)$ \\
\hline$>75$ & $9(4.2)$ \\
\hline \multicolumn{2}{|l|}{ Education } \\
\hline Illiterate & $21(9.8)$ \\
\hline End of elementary school & $51(23.7)$ \\
\hline End of middle school & $46(21.4)$ \\
\hline End of high school & $42(19.5)$ \\
\hline End of associate degree & $37(17.2)$ \\
\hline End of bachelor's degree & $15(7.0)$ \\
\hline End of master's degree & $1(0.5)$ \\
\hline PhD and more & $2(0.9)$ \\
\hline \multicolumn{2}{|l|}{ Occupation } \\
\hline Farmer & $12(5.6)$ \\
\hline Factory worker & $9(4.2)$ \\
\hline Receptionist & $1(0.5)$ \\
\hline Teacher & $5(2.3)$ \\
\hline University faculty & $3(1.4)$ \\
\hline Driver & $49(22.8)$ \\
\hline Office employee & $81(37.7)$ \\
\hline Home keeper & $21(9.8)$ \\
\hline Other & $34(15.8)$ \\
\hline
\end{tabular}

Abbreviation: SES, socio-economical status.

months.

2) I smoke but I seriously consider quitting smoking in the next 6 months. 
3) I smoke but I have decided to quit smoking in the next 30 days.

4) I am currently in the quitting status.

\subsection{Main Outcome}

Two questions measured lung cancer risk perception. During the data analysis process, these two questions were combined to create a new variable (Risk Perception score). This variable measures the overall risk perception and the scale range is between 0 and 20 .

\subsection{Study Size}

A sample size of 138 achieved $95 \%$ power to detect a correlation of 0.3 between the main outcomes of the study including lung cancer risk perception and self-efficacy (13), using a two-sided hypothesis test with a significance level of 0.05. Considering the design effect of the sampling procedure equals 1.5, the final sample size increased to 207 cases (24-26). Finally, a sample size of 215 was recruited.

\subsection{Statistical Analysis}

Mean (SD) and frequency (percent) were used for both numeric and categorical data. To assess the relationship between risk perception and underlying characteristics, simple and multiple general linear modeling was conducted. In the multiple general linear models, the relationships were assessed simultaneously and the effect of confounders was adjusted. The categorical variables were entered into the model as indicators. Spearman correlation was utilized to test the correlation between quitting intention and P-Sus, P-Sev, and Risk Perception score. In all analyses, P-values less than 0.05 were considered significant. The statistical analysis was conducted by SPSS software (Ver.17) (SPSS Inc. IL, Chicago, USA).

\section{Results}

\subsection{Participants}

Approximately 500 individuals were approached to assess their eligibility for this study. A total of 260 eligible participants entered the study. Finally, 215 participants were recruited in this study, among whom 192 (89.7\%) were current smokers.

\subsection{Descriptive Data}

The number of male participants was 185 (86.0\%) and the number of female participants was 30 (14.0\%). Information about the demographic characteristics and socioeconomic status of the participants was presented in Table 1. Minimum years of smoking were 1 and the maximum years of smoking were 68 with a mean of $30.77(S D=13.1)$. Most of the participants $(\mathrm{n}=199,92.5 \%)$ had a smoking history of 1 to 2 packs per day, and only 16 of them (7.4\%) smoked 2 to 4 packs per day.

\subsection{Outcome Data, Lung Cancer Risk Perception}

Analysis of the participants' responses to "P-Sus" and "P-Sev" was presented in Table 2. The mean of the lung cancer risk perception for P-Sus and P-Sev was 6.10 and 6.87 out of 10 and 12.97 out of 20 for Risk Perception score, respectively.

\begin{tabular}{lccc}
\hline \multicolumn{4}{l}{ Table 2. Analysis of the Responses to "P-Sus", "P-Sev", and Risk Perception Score ${ }^{\mathrm{a}}$} \\
\hline & P-Sus & P-Sev & Risk Perception Score \\
\hline Mean & 6.10 & 6.8744 & 12.9767 \\
Median & 7.00 & 7.0000 & 13.0000 \\
Standard deviation & 3.715 & 2.88859 & 5.07646 \\
Minimum & 0 & 0.00 & 0.00 \\
Maximum & 10 & 10.00 & 20.00 \\
\hline
\end{tabular}

Abbreviations: P-Sev, perceived severity; P-Sus, perceived susceptibility.

${ }^{\text {a }}$ Possible range for P-Sus and P-Sev is $(0-10)$ and for Risk Perception score is $(0$ -20).

4.4. Results of Modeling Risk Perception Variable Based on Background Variables

In this section, firstly, the relationship between risk perception with 5 background variables of (1) SES, (2) pack year, (3) age, (4) years smoked, and (5) sex was analyzed. This process is applied to the 3 variables related to risk perception; (1) P-Sus, (2) P-Sev, and (3) Risk Perception score.

\subsubsection{Modeling the Variable P-Sus with Background Variables}

The results of the modeling indicated that P-Sus was negatively related to "years smoked" in simple regression $(\mathrm{B}=-0.035, \mathrm{P}=0.069)$ and multiple regression $(\mathrm{B}=-0.069$, $\mathrm{P}=0.007)$. Moreover, the results of the modeling indicated that P-Sus was negatively related to the variable "age" only in the age category of 45 to 50 years $(\mathrm{B}=-1.981, \mathrm{P}=0.046)$. This result was not negatively related to the variable "age" in other age categories (51-55, 56 - 60, 61 - 65, 66+) (Table 3). 


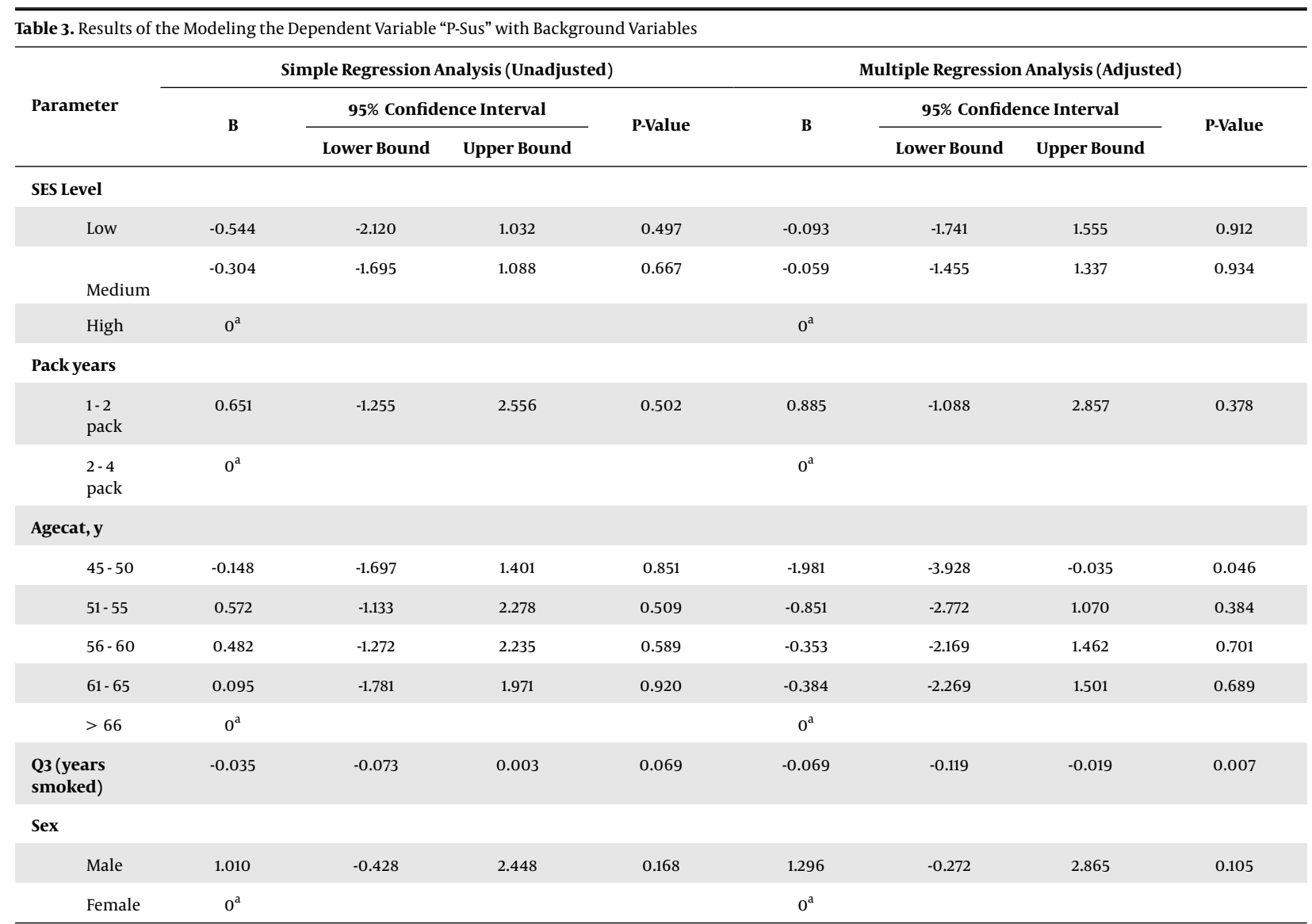

Abbreviations: Agecat, age category; P-Sus, perceived susceptibility.

${ }^{a}$ Reference category.

\subsubsection{Modeling the Variable P-Sev with Background Variables}

The results of the modeling indicated that P-Sev was negatively related to the variable "sex" $(\mathrm{B}=-1.037, \mathrm{P}=0.068)$. This negative relationship between "P-Sev" and "Sex" was present only among male participants (Table 4).

4.4.3. Modeling the Variable Risk Perception Score with Background Variables

The results of the modeling show no significant relationship between the variable Risk Perception Score and the background variables (Table 5). The results of the Spearman correlation indicated significant and direct correlations between "quitting intention" and P-Sus $(\mathrm{r}=0.296, \mathrm{P}$ $<0.001)$, P-Sev ( $\mathrm{r}=0.162, \mathrm{P}=0.009)$, and Risk Perception Score $(\mathrm{r}=0.315, \mathrm{P}<0.001)$.

\section{Discussion}

The main aim of this study was to investigate lung cancer risk perception among smokers. The findings indi- cated that lung cancer risk perception of smokers is moderately above the average. At the primary stage, this finding is crucial to the cancer prevention field because it explains individuals' perception of their lung cancer risk according to their cigarette-smoking behaviors (27). The relationship between risk perception and health behavior has extensively been explored in the past but the association between lung cancer risk perception among smokers is not clearly defined (27). There have been numerous research studies that have proposed causal relationships between lung cancer risk perception and cigarette-smoking behaviors in negative, positive, and no specific ways (28, 29). Moreover, some studies, instead of causality relations, have reported a positive correlation between lung cancer risk perception and the probability of smoking $(30,31)$. Other studies found different associations $(32,33)$. Future longitudinal studies could help to investigate this association in our population more deeply.

Intention to quit smoking is an indicator of a possible behavior change, justifying smokers' efforts to re- 


\begin{tabular}{|c|c|c|c|c|c|c|c|c|}
\hline \multirow{3}{*}{ Parameter } & \multicolumn{4}{|c|}{ Simple Regression Analysis (Unadjusted) } & \multicolumn{4}{|c|}{ Multiple Regression Analysis (Adjusted) } \\
\hline & \multirow{2}{*}{ B } & \multicolumn{2}{|c|}{ 95\% Confidence Interval } & \multirow{2}{*}{ P-Value } & \multirow{2}{*}{ B } & \multicolumn{2}{|c|}{ 95\% Confidence Interval } & \multirow{2}{*}{ P-Value } \\
\hline & & Lower Bound & Upper Bound & & & Lower Bound & Upper Bound & \\
\hline \multicolumn{9}{|l|}{ SES level } \\
\hline Medium & -0.410 & -1.488 & 0.668 & 0.454 & 0.562 & -1.658 & 0.534 & 0.313 \\
\hline High & $0^{\mathrm{a}}$ & & & & $0^{\mathrm{a}}$ & & & \\
\hline \multicolumn{9}{|l|}{ Pack years } \\
\hline $\begin{array}{l}1-2 \\
\text { pack }\end{array}$ & -0.001 & -1.484 & 1.482 & 0.999 & -0.318 & -1.866 & 1.231 & 0.686 \\
\hline \multicolumn{9}{|l|}{ Agecat, y } \\
\hline $45-50$ & 0.360 & -0.844 & 1.564 & 0.556 & 1.108 & -0.420 & 2.636 & 0.154 \\
\hline $51-55$ & -0.174 & -1.500 & 1.151 & 0.796 & 0.393 & -1.114 & 1.901 & 0.607 \\
\hline $56-60$ & -0.164 & -1.527 & 1.199 & 0.812 & 0.291 & -1.134 & 1.715 & 0.688 \\
\hline $61-65$ & -0.228 & -1.686 & 1.230 & 0.758 & -0.034 & -1.514 & 1.445 & 0.964 \\
\hline$>66$ & $0^{\mathrm{a}}$ & & & & $0^{\mathrm{a}}$ & & & \\
\hline $\begin{array}{l}\text { Q3 (years } \\
\text { smoked) }\end{array}$ & -0.001 & 0.015 & -0.055 & 0.957 & 0.018 & -0.021 & 0.057 & 0.362 \\
\hline \multicolumn{9}{|l|}{ Sex } \\
\hline Male & -1.037 & -2.151 & 0.078 & 0.068 & -1.031 & -2.263 & 0.200 & 0.100 \\
\hline
\end{tabular}

Abbreviations: Agecat, age category; P-Sus, perceived susceptibility.

${ }^{\text {a }}$ Reference category.

duce their risk to contract lung cancer. Quitting smoking, in general, can lower the risk of developing lung cancer regardless of age (34). Lung cancer risk perception among our participants is moderately above the average. This means that even though our target population views themselves at lung cancer risk, this perception is not high enough to make substantial behavioral changes to prevent them from the real risk of lung cancer. The results showed a significant correlation between quitting intention and Risk Perception Score, which is similar to the results of a study conducted by Ziebarth (35). In that study, it was concluded that smokers that do not plan to quit smoking are much more likely to underestimate their lifetime risk to develop lung cancer (35).

\subsection{P-Sus}

The results of this study show that the participants, who smoked for more years, have a lower risk perception of developing lung cancer compared to other participants. This contradicts findings of other studies such as a study conducted by Chen et al. (27) on a sample of 1,680 U.S. adults. In Chen et al.'s (27) study, it was discovered that lung cancer risk perception of the individuals who are engaged in cigarette-smoking behaviors is increased. Their findings suggested that laypeople may generally be conscious of the health hazard information of smoking. This information is dispersed through broad anti-smoking efforts and campaigns by the mass media, non-profit organizations, and the U.S. government (27). The lower risk perception of people who smoked more years in our study could be due to the lack of such educational programs and lower awareness about the health risk of smoking. We presume that these people are older too. On the other hand, in a study by Ziebarth (35), it is stated that when smokers smoke more cigarettes, it is less likely that they overestimate the actual risk to develop lung cancer.

Moreover, younger smokers ( 45 - 50 years) in our study had a lower lung cancer risk perception. This contradicts the findings of a study conducted by Viscusi (36) in 1991. This study concludes that risk perceptions are higher 


\begin{tabular}{|c|c|c|c|c|c|c|c|c|}
\hline \multirow{3}{*}{ Parameter } & \multicolumn{4}{|c|}{ Simple Regression Analysis (Unadjusted) } & \multicolumn{4}{|c|}{ Multiple Regression Analysis (Adjusted) } \\
\hline & \multirow{2}{*}{$\mathbf{B}$} & \multicolumn{2}{|c|}{ 95\% Confidence Interval } & \multirow{2}{*}{ P-Value } & \multirow{2}{*}{ B } & \multicolumn{2}{|c|}{ 95\% Confidence Interval } & \multirow{2}{*}{ P-Value } \\
\hline & & Lower Bound & Upper Bound & & & Lower Bound & Upper Bound & \\
\hline \multicolumn{9}{|l|}{ SES level } \\
\hline Low & -0.327 & -2.479 & 1.825 & 0.765 & -0.131 & -2.428 & 2.166 & 0.911 \\
\hline Medium & -0.714 & -2.615 & 1.186 & 0.460 & -0.621 & -2.567 & 1.325 & 0.530 \\
\hline High & $0^{\mathrm{a}}$ & & & & $0^{\mathrm{a}}$ & & & \\
\hline \multicolumn{9}{|l|}{ Pack years } \\
\hline $\begin{array}{l}1-2 \\
\text { pack }\end{array}$ & 0.650 & -1.955 & 3.255 & 0.623 & 0.567 & -2.182 & 3.316 & 0.685 \\
\hline $\begin{array}{l}2-4 \\
\text { pack }\end{array}$ & $0^{\mathrm{a}}$ & & & & $0^{\mathrm{a}}$ & & & \\
\hline \multicolumn{9}{|l|}{ Agecat, y } \\
\hline $45-50$ & 0.212 & -1.910 & 2.335 & 0.844 & -0.874 & -3.587 & 1.839 & 0.526 \\
\hline $51-55$ & 0.398 & -1.938 & 2.735 & 0.737 & -0.457 & -3.134 & 2.220 & 0.737 \\
\hline $56-60$ & 0.317 & -2.085 & 2.720 & 0.795 & -0.063 & -2.592 & 2.467 & 0.961 \\
\hline $61-65$ & -0.133 & -2.702 & 2.437 & 0.919 & -0.418 & -3.045 & 2.209 & 0.754 \\
\hline$>66$ & $0^{\mathrm{a}}$ & & & & $0^{\mathrm{a}}$ & & & \\
\hline $\begin{array}{l}\text { Q3 (years } \\
\text { smoked) }\end{array}$ & -0.036 & -0.088 & 0.016 & 0.173 & -0.051 & -0.120 & 0.019 & 0.150 \\
\hline \multicolumn{9}{|l|}{ Sex } \\
\hline Male & -0.027 & -2.001 & 1.947 & 0.978 & 0.265 & -1.921 & 2.451 & 0.811 \\
\hline Female & $0^{\mathrm{a}}$ & & & & $0^{a}$ & & & \\
\hline
\end{tabular}

Abbreviations: Agecat, age category.

${ }^{a}$ Reference category.

among younger age cohorts and younger smokers overestimate the overall lung cancer risks. These risk perceptions have consecutively a negative effect on smoking-related decisions (36). In other words, Viscusi (36) assumes that younger smokers engage in risky behaviors and continue smoking despite their high-risk perception. Viscusi (36) explains that a high level of risk perception among younger smokers is due to the widespread public dissemination of the smoking-related risk information in the past two decades and heightened social awareness. Since we have not measured the quitting intention of our participants according to their age categories, we cannot make any presumptions regarding risk perception and their quitting intention in our study. It is stated in the 2017 report by the American Cancer Society that smokers are more likely to underestimate the original risk of developing lung cancer compared to non-smokers (37). The results of this study also exhibit a significant correlation between quitting intention and P-Sus. This is also supported by Ziebarth's (35) study that smokers have a significantly lower lung cancer risk perception compared to non-smokers.

\subsection{P-Sev}

There is a widespread underestimation of lung cancer mortality, while, less than $20 \%$ of lung cancer patients survive 5 years after the diagnosis (35). The results revealed that male smokers ( $86.0 \%$ of our respondents) also had a lower perception of lung cancer survival. There is a lack of scientific studies comparing lung cancer risk perception of male and female smokers. However, referring to numerous studies conducted on overall risk perception of diseases and health awareness among women and men $(38,39)$, we can assume that higher risk perception among our female participants could be due to their overall higher health awareness. Moreover, smokers who are not in the status to quit smoking are considerably more inclined to underestimate this mortality risk (35). This is similar to our results, in which there was a significant correlation between quitting intention and P-Sev.

\subsection{Risk Perception Score}

The results of a study by Zibearth (35) confirmed the inclination of people to overestimate the lifetime risk of smokers contracting lung cancer. In Ziebarth's (35) study, nearly $50 \%$ of the participants overestimated this risk. However, $80 \%$ of the participants underestimated the deadliness of lung cancer while less than $20 \%$ of people who are diagnosed with lung cancer survive 5 years after the diagnosis. The results discovered no significant relationship between the variable Risk Perception Score and background variables. To successfully encourage smokers 
to quit smoking, future health promotion interventions, other than those that are focused on lung cancer risk perception (e.g. programs to educate the risk of other cancer types and health conditions associated with smoking) may be needed (27).

Lack of a control group to compare the perceived risk between smokers and non-smokers, gathering responses through a self-report system, and the cross-sectional nature of the study are limitations of this study. Another limitation was the unbalanced sample size in the subgroups, which may make the statistical tests under power for these subgroups and, hence, the results could be nonsignificant. Recruiting females were also challenging because smoking is a sensitive topic among women in this culture.

\subsection{Conclusions}

Participants' lung cancer risk perception is moderately above average. The results of modeling the risk perception based on background variables determined that participants who smoked more years and also younger participants (45 - 50 years) had a lower risk perception to develop lung cancer. Furthermore, male smokers had a lower perception of lung cancer survival compared to female smokers. Finally, the results discovered significant correlations between quitting intention and P-Sus, P-Sev, and Risk Perception score. These important findings could be utilized in program development for smoking cessation and promoting awareness about lung cancer. It could also help to design future studies to further investigate the underlying causes of these findings. Risk perception biases could be an excellent focus for public policy initiatives in supporting smokers in making more informed decisions.

\section{Acknowledgments}

The authors express acknowledgment and gratitude to Iran's National Elite Foundation, Center for International Science and Technology Cooperation (CISTC) for allocating funds to conduct this study. The authors would like to thank Tabriz University of Medical Sciences and the Department of Health for providing academic, logistics, and administrative support for this study. Special thanks go to Mr. Zeinali for his support in recruiting participants in Razi Hospital. We also express our gratitude to Dr. Leila Namvar(Pulmonologist) for her assistance in recruiting participants from the Pulmonology Clinic of Imam Reza Hospital. The authors also thank participants of this study for their time allocated to answer the survey questions.

\section{Footnotes}

Authors' Contribution: FZ did conceptualization, methodology, validation, investigation, resources, data curation, writing-original draft preparation, writing-review and editing, visualization, project administration, and funding acquisition. HA did conceptualization, methodology, writing-review, and editing. MAJ did conceptualization, methodology, software, validation, formal analysis, resources, data curation, writing-original draft preparation, writing-review and editing, visualization, and supervision.

Conflict of Interests: The authors have no conflict of interests.

Ethical Approval: The ethical approval code was IR.TBZMED.REC.1397.955.

Funding/Support: This study was supported by Iran's National Elites Foundation, Center for International Science and Technology Cooperation (CISTC).

Informed Consent: The study was anonymous and no identifying information was gathered from participants. Before the conduct of the questionnaire by the trained staff, the study's goals and objectives and participants' roles were explained to them and enough time was given to make a decision to participate. If they decided to answer the questions and be part of the study, then the staff proceeded further.

\section{References}

1. American Cancer Society. Key Statistics for Lung Cancer. 2017. Available from: https://www.cancer.org/cancer/non-small-cell-lung-cancer/ about/key-statistics.html.

2. Midthun DE. Early diagnosis of lung cancer. F1000Prime Rep. 2013;5:12. doi: 10.12703/P5-12. [PubMed: 23585930]. [PubMed Central: PMC3616602].

3. National Cancer Institute. Cancer of the Lung and Bronchus - SEER Stat Fact Sheets.2019, [cited 2019 Dec 3]. Available from: https://seer.cancer. gov/statfacts/html/lungb.html.

4. Ghasemian A, Rezaei N, Saeedi Moghaddam S, Mansouri A, Parsaeian M, Delavari A, et al. Tobacco Smoking Status and the Contribution to Burden of Diseases in Iran, 1990-2010: findings from the Global Burden of Disease Study 2010. Arch Iran Med. 2015;18(8):493-501. [PubMed: 26265517].

5. Rezaei S, Akbari Sari A, Arab M, Majdzadeh R, Mohammadpoorasl A Estimating Economic Burden of Cancer Deaths Attributable to Smoking in Iran.J Res Health Sci. 2015;15(4):228-33. [PubMed: 26728908].

6. Moosazadeh M, Ziaaddini H, Mirzazadeh A, Ashrafi-Asgarabad A, Haghdoost AA. Meta-analysis of Smoking Prevalence in Iran. Addict Health. 2013;5(3-4):140-53. [PubMed: 24494171].

7. O'Keeffe LM, Taylor G, Huxley RR, Mitchell P, Woodward M, Peters SAE. Smoking as a risk factor for lung cancer in women and men: a systematic review and meta-analysis. BMJ Open. 2018;8(10). e021611. doi: 10.1136/bmjopen-2018-021611. [PubMed: 30287668]. [PubMed Central: PMC6194454]. 
8. Alberg AJ, Nonemaker J. Who is at high risk for lung cancer? Population-level and individual-level perspectives. Semin Respir Crit Care Med. 2008;29(3):223-32. doi: 10.1055/s-2008-1076742. [PubMed: 18506660]. [PubMed Central: PMC3399915].

9. Alberg AJ, Samet JM. Epidemiology of lung cancer. Chest. 2003;123(1 Suppl):21S-49S. doi: 10.1378/chest.123.1_suppl.21s. [PubMed: 12527563].

10. Brown VJ. Risk perception: it's personal. Environ Health Perspect. 2014;122(10):A276-9. doi: 10.1289/ehp.122-A276. [PubMed: 25272337]. [PubMed Central: PMC4181910].

11. Fischhoff B, Bostrom A, Quadrel MJ. Risk perception and communication. Annu Rev Public Health. 1993;14:183-203. doi: 10.1146/annurev.pu.14.050193.001151. [PubMed: 8323585].

12. Gollust SE, Schroeder SA, Warner KE. Helping smokers quit: understanding the barriers to utilization of smoking cessation services. Milbank Q. 2008;86(4):601-27. doi: 10.1111/j.1468-0009.2008.00536.x. [PubMed: 19120982]. [PubMed Central: PMC2690372].

13. Birmingham WC, Hung M, Boonyasiriwat W, Kohlmann W, Walters ST, Burt RW, et al. Effectiveness of the extended parallel process model in promoting colorectal cancer screening. Psychooncology. 2015;24(10):1265-78. doi: 10.1002/pon.3899. [PubMed: 26194469]. [PubMed Central: PMC7161702].

14. Katapodi MC, Lee KA, Facione NC, Dodd MJ. Predictors of perceived breast cancer risk and the relation between perceived risk and breast cancer screening: a meta-analytic review. Prev Med. 2004;38(4):388402. doi: 10.1016/j.ypmed.2003.11.012. [PubMed: 15020172].

15. American Cancer Society. Lung Cancer Statistics, How Common is Lung Cancer. 2019, [cited 2020 Jan 7]. Available from: https://www.cancer. org/cancer/lung-cancer/about/key-statistics.html.

16. Peto J. That the effects of smoking should be measured in pack-years: misconceptions 4. Br J Cancer. 2012;107(3):406-7. doi: 10.1038/bjc.2012.97. [PubMed: 22828655]. [PubMed Central: PMC3405232].

17. Vandenbroucke JP, von Elm E, Altman DG, Gotzsche PC, Mulrow $\mathrm{CD}$, Pocock SJ, et al. Strengthening the Reporting of Observational Studies in Epidemiology (STROBE): explanation and elaboration. Int J Surg. 2014;12(12):1500-24. doi: 10.1016/j.ijsu.2014.07.014. [PubMed: 25046751].

18. Charkazi A, Shahnazi H, Ghourchaei AB, Mirkarimi K. Smoking behaviors in Iranian male students: An application of transtheoretical model. J Educ Health Promot. 2012;1:22. doi: 10.4103/2277-9531.99954. [PubMed: 23555125]. [PubMed Central: PMC3577395].

19. Jafari T, Shahrokhi S. Prevention, Treatment, and Control of Cigarett Use, Oil Industry Health Organization, Health management, Branch of Occupational and Family Health. Publication number: 294. 2010. Available from: http://nafass.old.piho.ir/UploadedFiles/XFiles/nafass/ pdf/dastor\%20ol\%20amal\%20ejrayee.pdf.

20. Soltanipour S, Heidarzadeh A, Jafarinezhad A. Reliability and validity of the Persian (Farsi) version of the Risk Perception Survey-Diabetes Mellitus. East Mediterr Health J. 2014;20(3):190-5. [PubMed: 24950077].

21. Wong NC, Cappella JN. Antismoking Threat and Efficacy Appeals: Effects on Smoking Cessation Intentions for Smokers with Low and High Readiness to Quit. J Appl Commun Res. 2009;37(1):1-20. doi: 10.1080/00909880802593928. [PubMed: 20046966]. [PubMed Central: PMC2680609].

22. Kotz D, Brown J, West R. Predictive validity of the Motivation To Stop Scale (MTSS): a single-item measure of motivation to stop smoking. Drug Alcohol Depend. 2013;128(1-2):15-9. doi: 10.1016/j.drugalcdep.2012.07.012. [PubMed: 22943961].

23. Chen LS, Kaphingst KA. Risk perceptions and family history of lung cancer: differences by smoking status. Public Health Genomics. 2011;14(1):26-34. doi: 10.1159/000294151. [PubMed: 20375490]. [PubMed Central: PMC3025884].

24. Graybill FA. An introduction to linear statistical models. 1. McGraw Hill, New York-Toronto-London;1961. 463 p. doi:10.1002/bimj.19610030412.

25. Guenther WC. Desk Calculation of Probabilities for the Distribution of the Sample Correlation Coefficient. Am Stat. 1977;31(1):45-8. doi: 10.1080/00031305.1977.10479195.

26. Zar JH. Biostatistical analysis. 2nd ed. Englewood Cliffs, N.J: PrenticeHall; 1984. 718 p.

27. Chen LS, Kaphingst KA, Tseng TS, Zhao S. How are lung cancer risk perceptions and cigarette smoking related?-testing an accuracy hypothesis. Transl Cancer Res. 2016;5(Suppl 5):S964-71. doi: 10.21037/tcr.2016.10.75. [PubMed: 29147644]. [PubMed Central: PMC5685517].

28. Finney Rutten LJ, Blake KD, Hesse BW, Augustson EM, Evans S. Illness representations of lung cancer, lung cancer worry, and perceptions of risk by smoking status. J Cancer Educ. 2011;26(4):747-53. doi: 10.1007/s13187-011-0247-6. [PubMed: 21688184]. [PubMed Central: PMC4251766].

29. Lundborg P, Lindgren B. Do They Know What They are Doing? Risk Perceptions and Smoking Behaviour Among Swedish Teenagers. J Risk Uncertainty. 2004;28(3):261-86. doi: 10.1023/b:risk.0000026098.84109.62.

30. Helweg-Larsen M, Nielsen GA. Smoking cross-culturally: risk perceptions among young adults in Denmark and the United States. Psychol Health. 2009;24(1):81-93. doi: 10.1080/08870440801932656. [PubMed: 20186641].

31. Weinstein ND, Marcus SE, Moser RP. Smokers' unrealistic optimism about their risk. Tob Control. 2005;14(1):55-9. doi: 10.1136/tc.2004.008375. [PubMed: 15735301]. [PubMed Central: PMC1747991].

32. Helweg-Larsen M, Stancioff LM. Acculturation matters: risk perceptions of smoking among Bosnian refugees living in the United States. J Immigr Minor Health. 2008;10(5):423-8. doi: 10.1007/s10903-007-91071. [PubMed: 18066716].

33. Liu JT, Hsieh CR. Risk perception and smoking behavior: Empirical evidence from Taiwan. J Risk Uncertainty. 1995;11(2):139-57. doi: 10.1007/bf01067682.

34. CDC-Division of Cancer Prevention and Control C. CDC - What Are the Risk Factors for Lung Cancer? Smoking; 2019, [cited 2018 Dec 3]. Available from: https://www.cdc.gov/cancer/lung/basic_info/risk_ factors.htm.

35. Ziebarth NR. Biased Lung Cancer Risk Perceptions: Smokers are Misinformed. Jahrbücher für Nationalökonomie und Statistik. 2018;238(5):395-421. doi: 10.1515/jbnst-2018-0017.

36. Viscusi WK. Age Variations in Risk Perceptions and Smoking Decisions. Rev Econ Stat. 1991;73(4):577-88. doi: 10.1515/jbnst-2018-0017.

37. American Cancer Society. Lifetime Risk of Developing or Dying From Cancer. 2018, [cited 2019 Nov 6]. Available from: https://www.cancer.org/cancer/cancer-basics/lifetime-probabilityof-developing-or-dying-from-cancer.html.

38. Katz RC, Meyers K, Walls J. Cancer awareness and self-examination practices in young men and women. J Behav Med. 1995;18(4):377-84. doi: 10.1007/BF01857661. [PubMed: 7500328].

39. Mitchell EW, Levis DM, Prue CE. Preconception health: awareness, planning, and communication among a sample of US men and women. Matern Child Health J. 2012;16(1):31-9. doi: 10.1007/s10995-0100663-y. [PubMed: 20734124] 